\title{
Lung Monophasic Synovial Sarcoma
}

National Cancer Institute

\section{Source}

National Cancer Institute. Lung Monophasic Synovial Sarcoma. NCI Thesaurus. Code C45633.

A monophasic synovial sarcoma arising from the lungs. 